\title{
Model-Based Control of a VSC-Based Power Generator with Synthetic Inertia Provision in an Isolated Micro-grid
}

\author{
F. $\operatorname{Hardan}^{1 *}$, R. Norman ${ }^{1}$, W. Leithead ${ }^{2}$ \\ ${ }^{1}$ School of Engineering, Newcastle University, Newcastle Upon Tyne, NE1 7RU, UK \\ ${ }^{2}$ Electronic \& Electrical Engineering Dept. University of Strathclyde, Glasgow G1 1XW, UK \\ *faysal.hardan@newcastle.ac.uk
}

\begin{abstract}
Distributed energy resources such as solar PV, wind and energy storage systems are increasingly being utilized within isolated and grid-connected microgrids. Their integration has prompted the move towards more advanced coordinated control for flexible, efficient and secure operation of microgrid-based power systems. This paper presents a new power control method based on a canonical inverse-model and implied synthetic inertia (SI), which is implemented on a VSC-based power generator (VSC-PG) for frequency response support. The control method augments a traditional power controller whilst the SI technique is blended with a new switching mechanism which enhances the performance of the VSC-PG when it is utilized with SI provision capability. The switching mechanism acts only when necessary, without contradicting the main frequency regulating sources. For assessment, the above method and SI technique have been designed and modelled for a 630kVA VSC-PG with DC-link batterybank. The VSC-PG was connected within an isolated/remote microgrid formed by an 11kV feeder, 1MVA diesel generator and variable load. The performances of the proposed power controller and SI provider have been tested and analysed using detailed Matlab/Simulink simulations and found to compare favourably with methods published in the literature.
\end{abstract}

\section{Introduction}

Integration of renewable power sources and energy storage systems into an electrical network can improve power supply availability whilst also increasing efficiency and reducing environmental emissions and running costs. In isolated microgrids, the storage systems, with other sources such as diesel engine generators (DEGs), can also ensure the continuous supply of electrical power in cases with limited fuel and low renewable power generation. However, such continuous power generation requires co-ordinated control which should consider all connected power sources, including the energy storage units, and other distributed energy sources that can connect to the DCside of the microgrid via voltage source converters (VSCs). Flexible dynamic power control and co-ordination between different modes of operation have been achieved using these power-electronic VSCs, equipped with efficient active and reactive power controllers [1] [2]. Several methods for direct power control have recently been reported in the literature for applications of grid-connection, which are based on VSCs [3][7]. The work reported in [3] and [4] demonstrated power control methods based on hysteresis controllers which act on power errors to select the switching-patterns of the VSC's power-electronic devices through look-up tables. Although the methods provide direct control for the network's active and reactive power, they generate a variable switching-frequency that is very difficult to manage at the converter output. The performance of hysteresis-based controllers is, in general, poor with respect to harmonic chatter. References [5] and [6] presented power control methods based on voltage and current variables in the stationary $\alpha-\beta$ frame, whilst reference [7] addressed the effect of unbalanced grid voltages on network currents using a power controller incorporating a very simple positive and negative sequence calculator that modifies references for the power control in order to obtain balanced grid currents.
The power control structure presented in this paper is based on a new canonical state-space model [8] formulated as a dynamic power-model. The controller structure incorporates the inverse power-model which inherently cancels out the effect of power component coupling. This control strategy was adopted because it achieves good power regulation without including power adjustment or control by a slow outer-loop around an inner current controller [9] [10]. Recent literature shows a continuing interest in hybrid ac/dc microgrids of various topologies and control methods for integrating distributed and renewable energy resources, including energy storage systems, into isolated and grid-connected electrical networks [11]-[13]. Paper [11] reviews configurations for dc/ac microgrids, providing comparisons and highlighting the features, with analysis for each, and suggesting further research regarding design and integration issues within power networks. One of the main requirements for these microgrids is the development of more complex control for their ac/dc networks and the interfaced VSC-based power sources, to include ancillary services such as frequency response [14], network voltage control, and gridconnection or islanded mode of operation [13]. Reference [12] proposes a novel and systematic way of modelling multi-vector energy sources in a microgrid, including an energy management method that is used to control operation with uncertainty of loads. An example of a coordinated control strategy for hybrid ac/dc microgrids with VSC and PV-wind-battery sources is reported in [1] with application in grid-connection and standalone operations. The authors proposed a model predictive power controller (MPPC) used for grid-connected mode and a model predictive voltage controller (MPVC) for islanded mode. However, microgrids with increasing levels of power electronicbased sources, decrease the available inertia of the power system due to the decreasing ratio of the total system kinetic energy with respect to the total power of the system [15]. Systems with reduced inertia may be susceptible to large frequency deviations 
beyond the permitted limits and a high rate of change of frequency (ROCOF), leading to system failure and power blackout due to the activation of protection devices [16]-[17]. Accordingly, such microgrids may be categorized as grids with low inertia, especially when they are islanded or isolated, operating with DEGs that have relatively low inertia constants compared with those of typical synchronous generators. Methods for providing a continuous synthetic inertia (SI) by exploiting the kinetic energy of wind turbine rotors with variable inertia constants or via energy storage systems, including VSCbased systems, were reported in [18] and [19] respectively. It has been reported in [19] that for a $d f / d t$ SI control method, a first-order lag with a large time-constant of around $1 \mathrm{~s}$ is required for a medium stiff point of common coupling (PCC) to the grid. Paper [20] reported a VSC that was controlled as a virtual synchronous generator using a power model with the mechanical model of a rotating generator and this resulted in emulation of the generator features. Using the orthogonality principle of the sinusoidal trigonometrical variables, as in the three-phase case, paper [21] developed a droop-based control strategy for a single-phase converter in an isolated microgrid.

This paper proposes a novel switching mechanism for syntheticinertia provision based on a power-controlled VSC, which enhances the frequency-response performance of a power system with rotating generators. The method is simulated and tested for an $11 \mathrm{kV}$ isolated microgrid supplied by diesel generators and compared with other methods reported in the literature. The paper also presents a newly developed powermodel, including analysis for interfacing the VSC to the AC grid, which is linearized for use in an enhanced model-based power controller. The model was utilized to determine the synthetic-inertia power-demand to support frequency stabilization of the microgrid.

The remainder of this paper is structured as follows: Section 2 of the paper presents the outline of a VSC-based power generator (VSC-PG) structure including its components and their integration. Section 3 covers aspects of the proposed power control method covering detailed modelling, analysis and design. Section 4 then discusses the concept of SI provision by the VSC-PG with analysis, design and realization procedure. In addition, an SI switching technique is proposed to enhance speed/frequency support for a DEG. Section 5 provides detailed simulation results which demonstrate the effectiveness of the proposed power control method and SI technique and demonstrate that their performance compares favourably to other methods and techniques reported in the literature. The conclusion of the reported research work is drawn in Section 6.

\section{The VSC-based power generator}

Battery energy storage, solar-photovoltaics and modern windturbine systems which include grid DC-links, utilize VSCs as connecting interfaces to the distribution networks. To demonstrate the performance of the proposed power control method and SI technique, a 630KVA VSC-PG with the components shown in Fig. 1 is considered. It is comprised of a 3-level, 3-phase PWM VSC (3L NPC-VSI), [22], [23], with a common DC-link, a delta/star transformer connected to a fourwire network and a harmonic filter. Voltage synthesis is achieved via control and switching functions embedded within the hardware of the VSC. Considering this VSC system as a power generator, the energy produced by various types of renewable and energy storage sources can be conditioned and delivered through the bipolar common DC-link. The selected 3level NPC structure of the VSC is naturally adapted to the

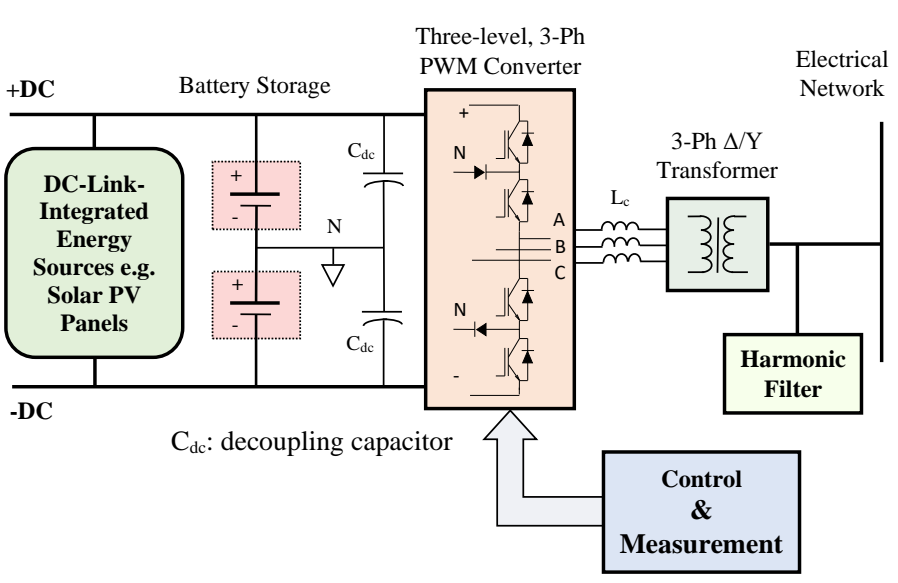

Fig. 1. Block diagram demonstrating the VSC-based PG

bipolar DC-link configuration, as it does not require balancing of the DC-link capacitors when compared with the five-level structure in [24].

\section{Power control via the VSC}

\subsection{Power representation for modelling and control}

In four-wire electrical networks (the case considered here), when the network three-phase voltages are balanced, the instantaneous voltages may be substituted with their $\mathrm{d}$ and $\mathrm{q}$ equivalent components within the orthogonal synchronous frame [25]. In this case, the active and reactive powers that are delivered or absorbed at the PCC can be given in their instantaneous power-invariant forms, [26], as,

$P_{n}=\mathcal{R}_{e}\left(\overrightarrow{\boldsymbol{v}}_{n d q} \cdot \overrightarrow{\boldsymbol{\imath}}_{n d q}^{*}\right)$,

$Q_{n}=-I_{m}\left(\overrightarrow{\boldsymbol{v}}_{n d q} \cdot \overrightarrow{\boldsymbol{i}}_{n d q}^{*}\right)$,

$P_{n}=f_{P}\left(v_{n d}, i_{n d}, v_{n q}, i_{n q}, t\right)=v_{n d} i_{n d}+v_{n q} i_{n q}$,

$Q_{n}=f_{Q}\left(v_{n d}, i_{n q}, v_{n q}, i_{n d}, t\right)=v_{n d} i_{n q}-v_{n q} i_{n d}$,

where, $\overrightarrow{\boldsymbol{v}}_{n d q}=v_{n d}+j v_{n q}, \overrightarrow{\boldsymbol{i}}_{n d q}=i_{n d}+j i_{n q}$ and $\mathcal{R}_{e}, I_{m}$ indicate the real and the imaginary parts of the resulting vectors respectively, $*$ denotes the conjugate vector, and $f_{P}, f_{Q}$ are representative functions. It is assumed that reactive power flowing into the load or towards the relevant node is negative, resulting in the "-" sign for $Q_{n}$. However, as the control voltages produced are of PWM form, they lead to output currents that contain a considerable number of harmonics. Accordingly, calculations of instantaneous active and reactive power are affected. These harmonics and their effect on power measurement can be reduced by employing a higher PWM switching frequency for the converter's switching devices. For the work reported in this paper, the converter is designed to operate at a $5250 \mathrm{~Hz}$ PWM switching frequency; that is a trade- 
off between shorter sampling-interval and acceptable switching power losses for high power applications.

\subsection{The power network-connection model and analysis}

Considering that control is only implemented by varying the fundamental or average voltage component, a power state-space model can be derived as follows. From (2), the derivatives of active and reactive power with respect to time can be given as,

$\frac{d P_{n}}{d t}=\frac{\partial f_{P}}{\partial v_{n d}} \frac{d v_{n d}}{d t}+\frac{\partial f_{P}}{\partial i_{n d}} \frac{d i_{n d}}{d t}+\frac{\partial f_{P}}{\partial v_{n q}} \frac{d v_{n q}}{d t}+\frac{\partial f_{P}}{\partial i_{n q}} \frac{d i_{n q}}{d t}$,

$=i_{n d} \frac{d v_{n d}}{d t}+v_{n d} \frac{d i_{n d}}{d t}+i_{n q} \frac{d v_{n q}}{d t}+v_{n q} \frac{d i_{n q}}{d t}$,

$\frac{d Q_{n}}{d t}=\frac{\partial f_{Q}}{\partial v_{n d}} \frac{d v_{n d}}{d t}+\frac{\partial f_{Q}}{\partial i_{n q}} \frac{d i_{n q}}{d t}+\frac{\partial f_{Q}}{\partial v_{n q}} \frac{d v_{n q}}{d t}+\frac{\partial f_{Q}}{\partial i_{n d}} \frac{d i_{n d}}{d t}$,

$=i_{n q} \frac{d v_{n d}}{d t}+v_{n d} \frac{d i_{n q}}{d t}-i_{n d} \frac{d v_{n q}}{d t}-v_{n q} \frac{d i_{n d}}{d t} ;$

When the d-axis of the synchronous frame is aligned with the real axis of the complex plane, which is usually the case, $v_{n q}=$ 0 , leading to $d v_{n q} / d t=0$, and $P_{n}=v_{n d} i_{n d}, Q_{n}=v_{n d} i_{n q}$; $v_{n d}=V_{n}$, which is equal to the line-voltage $V_{l l}$ at the PCC for power-invariant d-q frame representation. Denoting $d v_{n d} / d t$ as $v_{n d}^{\prime}=\grave{V}_{n},(3)$ and (4) can be rewritten as,

$\frac{d P_{n}}{d t}=v_{n d} \frac{d i_{n d}}{d t}+v_{n q} \frac{d i_{n q}}{d t}+P_{n} \frac{v_{n}}{v_{n}}$

$\frac{d Q_{n}}{d t}=v_{n d} \frac{d i_{n q}}{d t}-v_{n q} \frac{d i_{n d}}{d t}+Q_{n} \frac{\vec{v}_{n}}{V_{n}}$.

In general, when the electrical network is relatively voltage-stiff at the PCC voltage variations can be considered slow and the terms in $\grave{V}_{n}$ can be neglected.

From a control system point of view, active and reactive power controllers must produce an output voltage-vector that can be synthesized to three-phase PWM signals by a PWM modulator, which determines the switching instances of the powerelectronic devices of the VSC. The converter's 3-phase arms are connected to the electrical network through a 3-phase linking inductance and the $\Delta / Y$ (Delta/Star) transformer. Accordingly, the network-connection model, as shown in Fig. 2(a), must consider the parameters of both the inductance and the transformer. It is appropriate to neglect the effect of the magnetizing components of the connection transformer and to only include its winding transient inductances and resistances. By transferring the transient impedance of the transformer's $Y$ windings to its $\Delta$ (or primary) windings (taking into consideration the $\Delta / Y$ conversion) and adding the results to the primary impedance and the linking impedance, a total impedance $R_{n}+j \omega_{e} L_{n}$ can be obtained which represents a simplified electrical model for network-connection, as shown in Fig. 2(b). From this circuit model, the current derivative terms of (5) and (6) can be obtained as,

$\frac{d i_{n d}}{d t}=-\frac{R_{n}}{L_{n}} i_{n d}+\omega_{e} i_{n q}+\frac{1}{L_{n}} D v_{i d}$,

$\frac{d i_{n q}}{d t}=-\frac{R_{n}}{L_{n}} i_{n q}-\omega_{e} i_{n d}+\frac{1}{L_{n}} D v_{i q}$, where, $D v_{i d}$ and $D v_{i q}$ represent the $\mathrm{d}$ and $\mathrm{q}$ components of the input voltage vector $D \overrightarrow{\boldsymbol{v}}_{i d q}$ in the d-q synchronous frame, and $\omega_{e}$ is the angular frequency of the network voltage in $\mathrm{rad} / \mathrm{s}$.

Substituting for $d i_{n d} / d t, d i_{n q} / d t, i_{n d}$ and $i_{n q}$ from (7), (8) and (2) into (5) and (6), the final result is:

$\frac{d P_{n}}{d t}=\left(\frac{\grave{V_{n}}}{V_{n}}-\frac{R_{n}}{L_{n}}\right) P_{n}+\omega_{e} Q_{n}+\frac{1}{L_{n}} v_{n d} D v_{i d}+\frac{1}{L_{n}} v_{n q} D v_{i q}$,

$\frac{d Q_{n}}{d t}=\left(\frac{V_{n}}{V_{n}}-\frac{R_{n}}{L_{n}}\right) Q_{n}-\omega_{e} P_{n}-\frac{1}{L_{n}} v_{n q} D v_{i d}+\frac{1}{L_{n}} v_{n d} D v_{i q}$.

Equations (9) and (10) can be arranged in the form of a power state-space model as,

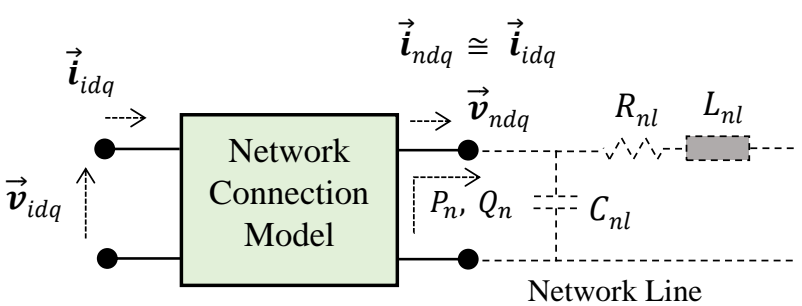

(a)

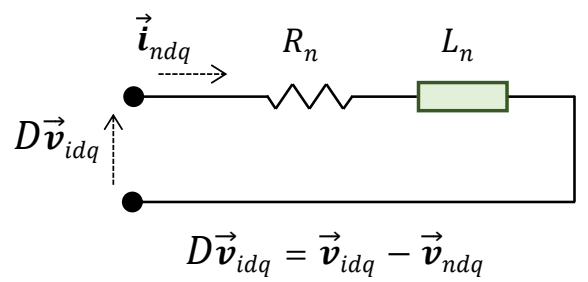

(b)

Fig. 2. The simplified network-connection model as an equivalent circuit

$\left[\begin{array}{c}\frac{d P_{n}}{d t} \\ \frac{d Q_{n}}{d t}\end{array}\right]=\left[\begin{array}{cc}\frac{\grave{V_{n}}}{V_{n}}-\frac{1}{\tau_{n}} & \omega_{e} \\ -\omega_{e} & \frac{V_{n}}{V_{n}}-\frac{1}{\tau_{n}}\end{array}\right]\left[\begin{array}{l}P_{n} \\ Q_{n}\end{array}\right]+\left[\begin{array}{cc}\frac{v_{n d}}{L_{n}} & \frac{v_{n q}}{L_{n}} \\ -\frac{v_{n q}}{L_{n}} & \frac{v_{n d}}{L_{n}}\end{array}\right]\left[\begin{array}{l}D v_{i d} \\ D v_{i q}\end{array}\right]$,

or in its symbolic form,

$\grave{\boldsymbol{S}_{n}}=\boldsymbol{A} \boldsymbol{S}_{n}+\boldsymbol{B} \boldsymbol{U}_{i d q}$,

where, $\tau_{n}=L_{n} / R_{n}$ is the time-constant of the networkconnection system. The model outputs are the active and reactive power delivered to the network, which can be regulated by applying the corresponding input voltage components, $D v_{i d}$, $D v_{i q}$. This system model is linear parameter-varying (LPV) [27] as its transient matrix, $\boldsymbol{A}$, and the input matrix, $\boldsymbol{B}$, depend on time-varying exogenous variables, respectively, the network's angular frequency $\omega_{e}$ and the network voltage components. Linearizing (12) relative to the frequency-voltage operatingpoint or OP (usually the nominal frequency and PCC voltage) using the Taylor series expansion, the perturbation power model can be formulated as, 
$\Delta \grave{\boldsymbol{S}}_{n} \approx \boldsymbol{A}^{o p} \Delta \boldsymbol{S}_{n}+\boldsymbol{B}^{o p} \Delta \boldsymbol{U}_{i d q}$.

In this case, the frequency at the PCC represents the stiff frequency of the power system under grid-connection mode, and is substituted for the electrical speed of the DEG in islanded mode. In both modes $\omega_{e}$ can be a slow-varying mechanical variable (due to the generators' moment of inertia) in comparison with the electrical or electromagnetic power, and the voltage at the PCC is considered to be a constant or slowly changing variable. The exogenous variables, thus vary slowly and the model can equally well be linearized for control purposes by freezing the exogenous variables [28] when

$\grave{\boldsymbol{S}_{n}} \approx \boldsymbol{A}^{o p} \boldsymbol{S}_{n}+\boldsymbol{B}^{o p} \boldsymbol{U}_{i d q}$.

Accordingly, Equation (14) at the nominal OP can be utilized for frequency analysis by expressing the power model in the Laplace or frequency domain as,

$\left[\begin{array}{l}P_{n}(s) \\ Q_{n}(s)\end{array}\right]=\left[\begin{array}{ll}g_{11}(s) & g_{12}(s) \\ g_{21}(s) & g_{22}(s)\end{array}\right]\left[\begin{array}{l}D v_{i d}(s) \\ D v_{i q}(s)\end{array}\right]=\boldsymbol{G}(s)\left[\begin{array}{c}D v_{i d}(s) \\ D v_{i q}(s)\end{array}\right]$;

$g_{11}(s)=\frac{\left(s+\left(\frac{1}{\tau_{n}}-\frac{\grave{V_{n}}}{V_{n}}\right)\right) \frac{v_{n d}}{L_{n}}-\frac{\omega_{e} v_{n q}}{L_{n}}}{\left(s+\left(\frac{1}{\tau_{n}}-\frac{V_{n}}{V_{n}}\right)\right)^{2}+\omega_{e}^{2}}$,

$g_{12}(s)=\frac{\left(s+\left(\frac{1}{\tau_{n}}-\frac{V_{n}}{V_{n}}\right)\right) \frac{v_{n q}}{L_{n}}+\frac{\omega_{e} v_{n d}}{L_{n}}}{\left(s+\left(\frac{1}{\tau_{n}}-\frac{V_{n}}{V_{n}}\right)\right)^{2}+\omega_{e}^{2}}$,

$g_{21}(s)=-g_{12}(s), \quad g_{22}(s)=g_{11}(s)$.

From the above system transfer function, it can be found that the system has two conjugate poles with decaying and oscillatory properties,

$P_{1,2}=\left\{\begin{array}{l}-\left(\frac{1}{\tau_{n}}-\frac{\grave{V_{n}}}{V_{n}}\right)+j \omega_{e} \\ -\left(\frac{1}{\tau_{n}}-\frac{\grave{V_{n}}}{V_{n}}\right)-j \omega_{e}\end{array}\right.$,

By considering the diagonal elements of the transient matrix $\boldsymbol{A}$ or the eigenvalues, $P_{1,2}$, it can be determined that the model is exponentially stable, i.e. the eigenvalues are in the left half of the complex plane, as $\tau_{n}$ is always positive, unless $\grave{V}_{n} / V_{n}$ exceeds the value of $1 / \tau_{n}$. However, $\grave{V}_{n} / V_{n}$ would be sufficiently small (much lower than $1 / \tau_{n}$ ) for a relatively voltage-stiff PCC. The other cross-elements of matrix $\boldsymbol{A}$, produce an oscillatory response at the electrical angular frequency $\omega_{e}$. Considering that the synthetic inertia via the VSC, like other additional rotating generators added to the microgrid, will support the stabilization of this frequency and the grid voltage, it is acceptable to consider that the frequency is constant (or updated as a slow variable) and that the term $\grave{V}_{n} / V_{n}$ is negligible throughout the modelling and analysis presented in this paper. Further research to investigate the effect of model non-linearity due to voltage and frequency variations is beyond the scope of the paper.
Substituting the values for $\omega_{e}$ and $\tau_{n}$ from Appendix 1, the poles will have the values: $P_{1}=-16+j 314.16, P_{2}=-16-$ $j$ 314.16. Changes to the components of matrix $\boldsymbol{A}$ influence the position of the model's poles, whilst changes in matrix $\boldsymbol{B}$ components (or the network's voltage magnitude) affect the gains of the model transfer functions in (15).

On discretising the model, provided that the sampling-interval is sufficiently small, the changes in $\boldsymbol{A}$ and $\boldsymbol{B}$ between two consecutive updates are very small and the model in (12) can be updated continuously for the current OP. Updating $\omega_{e}$ within $\boldsymbol{A}$ will move the model to a slightly different stable operating-point with a slightly different oscillation frequency.

\subsection{Conventional direct power control}

Based on the power model above, a conventional power controller (CPC) can be built, as demonstrated in Fig. 3. $\mathrm{C}_{\mathrm{p}}(\mathrm{s})$ and $\mathrm{C}_{\mathrm{q}}(\mathrm{s})$ are the active and reactive power controllers respectively.

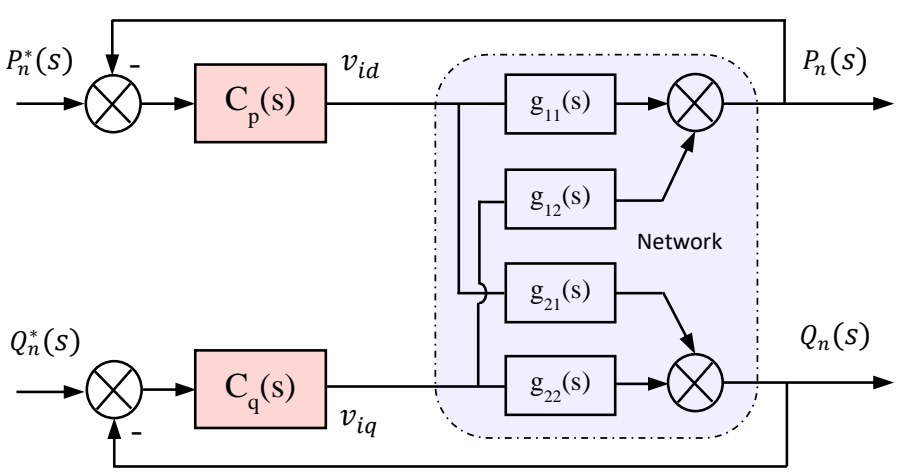

Fig. 3. The structure of the conventional power controller

From the structure given in Fig. 3, the power closed-loop transfer functions can be written as,

$$
\begin{aligned}
& F_{p c}(s)=\frac{P_{n}(s)}{P_{n}^{*}(s)}=\frac{C_{p} g_{11}+C_{q} C_{p} g_{22} g_{11}-C_{p} C_{q} g_{21} g_{12}}{1+C_{q} g_{22}+C_{p} g_{11}+C_{q} C_{p} g_{22} g_{11}-C_{p} C_{q} g_{21} g_{12}}, \\
& F_{q c}(s)=\frac{Q_{n}(s)}{Q_{n}^{*}(s)}=\frac{C_{q} g_{22}+C_{q} C_{p} g_{22} g_{11}-C_{q} C_{p} g_{12} g_{21}}{1+C_{p} g_{11}+C_{q} g_{22}+C_{q} C_{p} g_{22} g_{11}-C_{q} C_{p} g_{12} g_{21}}, \\
& F_{p q c}(s)=\frac{P_{n}(s)}{Q_{n}^{*}(s)}=\frac{C_{q} g_{12}}{1+C_{p} g_{11}+C_{q} g_{22}+C_{q} C_{p} g_{22} g_{11}-C_{q} C_{p} g_{12} g_{21}}, \\
& F_{q p c}(s)=\frac{Q_{n}(s)}{P_{n}^{*}(s)}=\frac{C_{p} g_{21}}{1+C_{q} g_{22}+C_{p} g_{11}+C_{q} C_{p} g_{22} g_{11}-C_{p} C_{q} g_{21} g_{12}} ;
\end{aligned}
$$

where the Laplace operator '(s)' for all $g_{m n}(s)$ functions and $C_{p}(s), C_{q}(s)$ is omitted for convenience. To have symmetrical responses for the active and reactive power, $C_{p}(s), C_{q}(s)$ are set to be identical. From the above closed-loop functions, controllers $C_{p}(s)$ or $C_{q}(s)$ can be determined, using various frequency-domain methods based on the defined performance specification in [29]-[30]. However, for demonstration purposes 
in this paper, $C_{p}(s), C_{q}(s)$ have been designed following the simple structure of the PI control action with the following transfer function,

$C_{p}(s)=C_{q}(s)=\frac{K_{p} s+K_{i}}{s}$.

The control design process is now to find the optimum values of $K_{p}$ and $K_{i}$ such that the main closed-loop frequency responses $F_{p c}(s), F_{q c}(s)$ are as close as possible to unity over a wide frequency band from DC, while reducing as much as possible the unfavourable cross-coupling frequency responses $F_{p q c}(s)$ and $F_{q p c}(s)$. This process ensures the best possible reference or demand following for the output active and reactive power. Increasing both $K_{p}$ and $K_{i}$ widens the control bandwidth whilst gains at low frequencies and rejection of steady-state errors are mainly increased by increasing the value of $K_{i}$. Using (16) to (19) for analysis and controller design, different step-responses can be obtained as shown in Fig. 4 with fixed $K_{p}=0.001$ and different values of $K_{i}$.

It is clear that the step-response is sensitive to the parameter $K_{i}$. When the response is oscillatory (e.g. for $K_{i}=5$ and 1) crosscoupling effects vanish quickly whilst for a better response shape (e.g. $K_{i}=0.05$ ) cross-coupling effects last considerably longer. Therefore, a trade-off between a smoother power stepresponse and shorter cross-coupling effects has to be adopted in this case, which proves to be quite demanding. This dilemma has been overcome by utilizing the control method presented in the next section.

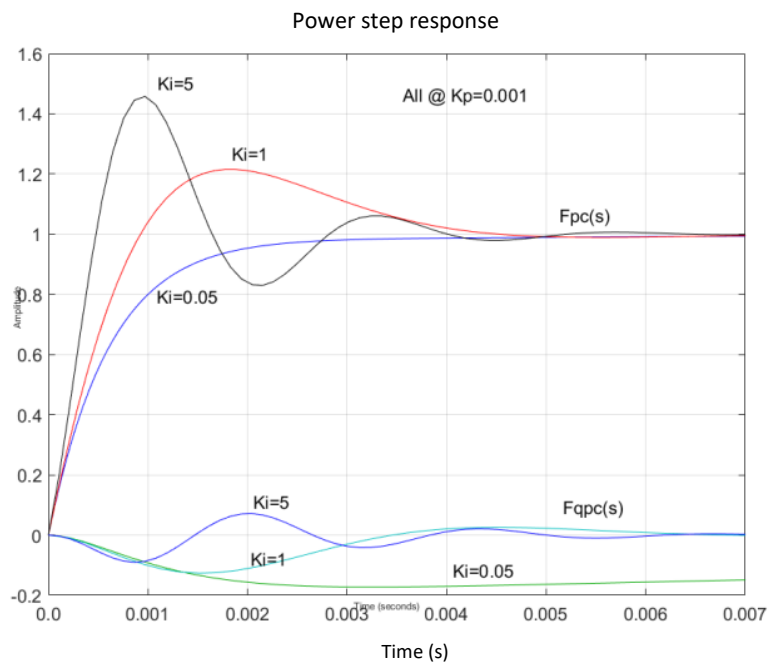

Fig. 4. Step-responses of conventional power controllers

\subsection{The proposed inverse-model power controller}

3.4.1. The concept of the proposed control method: Theoretically, an inverse-model power controller (IMPC) could be implemented to provide satisfactory open-loop control within a practical bandwidth assuming that the model is accurate. However, the power model derived above is not perfectly matched with the real physical system, and voltage measurement for the model contains noise at synchronous and PWM frequencies that cannot be fully eliminated. Therefore, a closed-loop control structure, augmented with a power inversemodel, is proposed as shown in Fig. 5 [31]. The connection of the inverse-model $\boldsymbol{G}^{-1}(s)$ in series with the physical network $\boldsymbol{G}_{p}(s)$ will internally reduce any cross-coupling between the control channels of the active and reactive power. From Fig. 5, the multi-input, multi-output (MIMO) closed-loop transfer function from $\boldsymbol{S}_{n}^{*}(s)$ to $\boldsymbol{S}_{n}(s)$ can be found as,

$\boldsymbol{F}_{c l}(s)=\boldsymbol{S}_{n}^{*-1}(s) \boldsymbol{S}_{n}(s)$,

$=\left[\boldsymbol{G}^{-1}(s) \boldsymbol{G}_{p}(s)+\boldsymbol{C}(s) \boldsymbol{G}_{p}(s)\right]\left[\boldsymbol{I}+\boldsymbol{T}(s) \boldsymbol{C}(s) \boldsymbol{G}_{p}(s)\right]^{-1}$.

where, $\boldsymbol{C}(s)=\operatorname{diag}\left[C_{p}(s) C_{q}(s)\right], \boldsymbol{T}(s)$ is the transfer function of the measurement transducers and $\boldsymbol{I}$ is the identity matrix. When $\boldsymbol{G}(s)$ is closely matched to $\boldsymbol{G}_{p}(s)$ and $\boldsymbol{T}(s)$ is equal to the identity matrix for accurate measurements, then $\boldsymbol{F}_{c l}(s)$ becomes:

$\boldsymbol{F}_{c l}(s) \approx\left[\boldsymbol{I}+\boldsymbol{C}(s) \boldsymbol{G}_{p}(s)\right]\left[\boldsymbol{I}+\boldsymbol{C}(s) \boldsymbol{G}_{p}(s)\right]^{-1}=\boldsymbol{I}$.

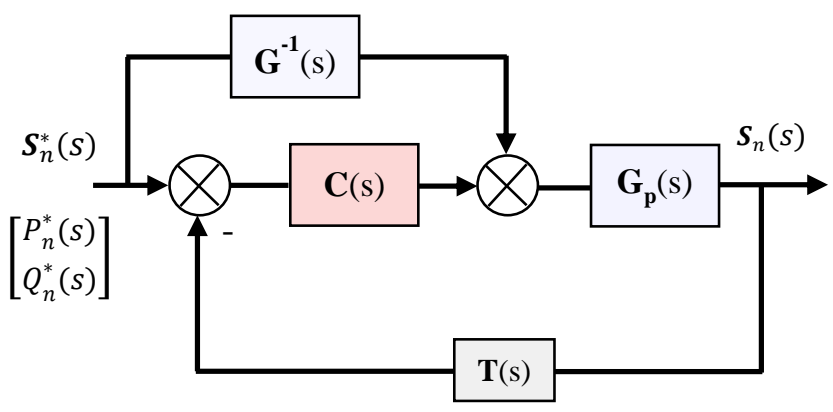

Fig. 5. The closed-loop control structure augmented with the inverse-model

This result demonstrates that the closed-loop frequency response is almost ideal and can be equal to unity for a precise model when $\boldsymbol{G}(s)=\boldsymbol{G}_{p}(s)$. The unity closed-loop response guarantees that the output apparent power $\boldsymbol{S}_{n}$ follows the demand power $\boldsymbol{S}_{n}^{*}(s)$ precisely without distortions or delays. Clearly the above result can be achieved regardless of the controller, $\boldsymbol{C}(s)$. By virtue of the feedback power loop, the controller can be tuned to eliminate any control errors that may arise due to deviation of model parameters and due to any unmodelled network dynamics.

3.4.2. Realization of the inverse-model power controller: Based on the above inverse-model control method, the structure of the proposed IMPC can be illustrated in terms of two power channels, as shown in Fig. 6. From (15), as $v_{n q}=0$, the frequency-domain inverse-model of the network-connection power model becomes, 
$\left[\begin{array}{c}D v_{i d}(s) \\ D v_{i q}(s)\end{array}\right] \cong\left[\begin{array}{cc}\frac{L_{n}}{v_{n d}}\left(s+\frac{1}{\tau_{n}}\right) & -\frac{\omega_{e} L_{n}}{v_{n d}} \\ \frac{\omega_{e} L_{n}}{v_{n d}} & \frac{L_{n}}{v_{n d}}\left(s+\frac{1}{\tau_{n}}\right)\end{array}\right]\left[\begin{array}{c}P_{n}(s) \\ Q_{n}(s)\end{array}\right]=$

$\boldsymbol{G}(s)^{-1}\left[\begin{array}{l}P_{n}(s) \\ Q_{n}(s)\end{array}\right]$.

The inclusion of this power inverse-model $\boldsymbol{G}^{-1}(s)$ within the controller structure, inherently provides power de-coupling control, and as a complete inverse-model, it results in the functions $C_{p}(s)$ and $C_{q}(s)$ becoming as simple as possible, only tuned to compensate for steady-state errors and, importantly, to help in suppressing power oscillation. For performance comparison, the same PI controllers used in section 3.3 have been considered here within the inverse-model control structure as illustrated in Fig. 6. The controller's pole provides the DC error rejection whilst its zero is adjusted for rejecting highfrequency errors at the bandwidth of interest. The inverse-model was implemented in its discrete form, with the sampling rate chosen to equal the switching frequency, $5250 \mathrm{~Hz}$. However, as the rise-time of the closed-loop power step-response for the continuous-time model is around $1 \mathrm{~ms}$, its sampling rate is not high enough to use standard discretisation transformations such as the forward difference method. Therefore, a more accurate discrete representation has been used as follows,

$\boldsymbol{S}_{n}((k+1) T)=\boldsymbol{G}(T) \boldsymbol{S}_{n}(k T)+\boldsymbol{H}(T) \boldsymbol{U}_{n}(k T) ;$

$\boldsymbol{S}_{n}(k T)=\left[\begin{array}{c}P_{n}(k T) \\ Q_{n}(k T)\end{array}\right], \boldsymbol{U}_{n}(k T)=\left[\begin{array}{c}D v_{i d}(k T) \\ D v_{i q}(k T)\end{array}\right]$,

$\boldsymbol{G}(T)=\boldsymbol{e}^{A T}=\left[\begin{array}{ll}G_{11}(T) & G_{12}(T) \\ G_{21}(T) & G_{22}(T)\end{array}\right]$,

$\boldsymbol{H}(T)=\boldsymbol{A}^{-1}(\boldsymbol{G}(T)-\boldsymbol{I}) \boldsymbol{B}=\left[\begin{array}{ll}H_{11}(T) & H_{12}(T) \\ H_{21}(T) & H_{22}(T)\end{array}\right]$

where, $T$, is the sampling period and $k$, is the sample order. Using the resolvent method, [8], $\boldsymbol{e}^{\boldsymbol{A T}}$ or $\boldsymbol{G}(T)$ can be found as,

$\boldsymbol{G}(T)=\left[\begin{array}{cc}e^{\frac{-T}{\tau_{n}}} \cos \left(\omega_{e} T\right) & e^{\frac{-T}{\tau_{n}}} \sin \left(\omega_{e} T\right) \\ -e^{\frac{-T}{\tau_{n}}} \sin \left(\omega_{e} T\right) & e^{\frac{-T}{\tau_{n}}} \cos \left(\omega_{e} T\right)\end{array}\right]$.

Accordingly, the discrete-time power inverse-model is,

$\left[\begin{array}{c}D v_{i d}^{*}(k T) \\ D v_{i q}^{*}(k T)\end{array}\right]=\left[\begin{array}{ll}H_{11}(T) & H_{12}(T) \\ H_{21}(T) & H_{22}(T)\end{array}\right]^{-1}\left\{\left[\begin{array}{l}P_{n}^{*}((k+1) T) \\ Q_{n}^{*}((k+1) T)\end{array}\right]-\right.$

$\left.\left[\begin{array}{ll}G_{11}(T) & G_{12}(T) \\ G_{21}(T) & G_{22}(T)\end{array}\right]\left[\begin{array}{l}P_{n}(k T) \\ Q_{n}(k T)\end{array}\right]\right\}$, which produces the main control voltage components $D v_{i d}^{*}$, $D v_{i q}^{*}$, as demonstrated in Fig. 6. The inverse-model, (25), estimates the required input voltage-differences, $\boldsymbol{U}_{n}^{*}(k T)$, to achieve $\boldsymbol{S}_{n}^{*}((k+1) T)$, i.e. targets for $P_{n}^{*}$ and $Q_{n}^{*}$ set one step ahead by the reference inputs to the controller, following a similar philosophy to general model predictive control (MPC). For most of the time, the reference inputs will remain unchanged or will only change slowly, but when rapid or large changes are required, the $P_{n}^{*}$ and $Q_{n}^{*}$ demands must be moderated by prefilters to prevent the changes in them from being too large. It should be noted that Fig. 6 represents a mixed signal diagram and for consistency with Fig. 5, the inputs to these functions are $P_{n}^{*}(k T) \& P_{n}(k T)$ and $Q_{n}^{*}(k T) \& Q_{n}(k T)$ whereas the inputs to the inverse model are $P_{n}^{*}((k+1) T)$ and $Q_{n}^{*}((k+1) T)$.

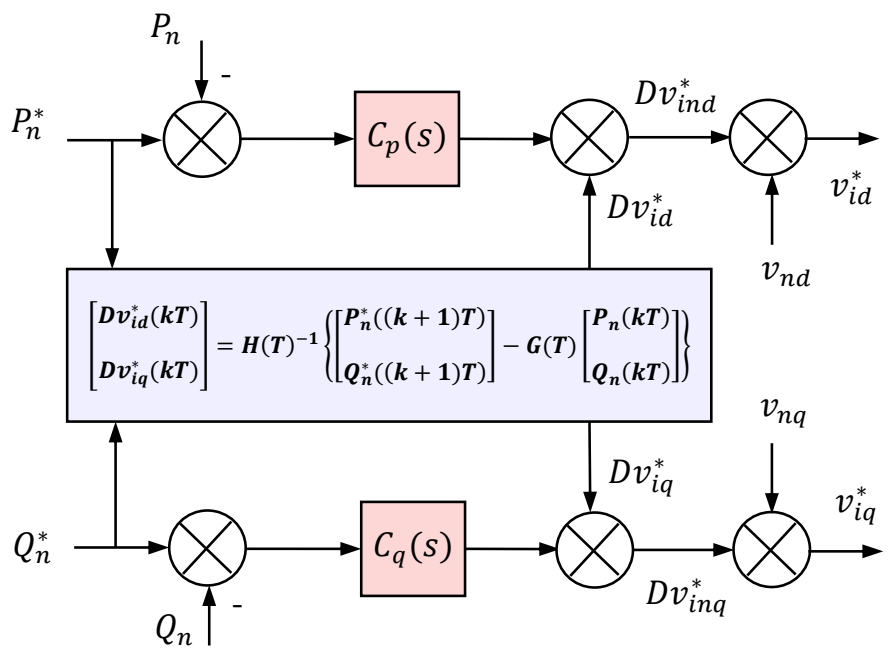

Fig. 6. Realization block diagram of the proposed inverse-model power controller

The PI control functions, $C_{p}(s)$ and $C_{q}(s)$, generate control voltage components which compensate for active and reactive power-errors that may arise due to modelling inaccuracy. These components are added to the inverse-model voltage components to generate the voltage control signals $D v_{i n d}^{*}, D v_{i n q}^{*}$ for the $\mathrm{d}$ and $q$ channels. At the final stage, the reference $d$ and $q$ voltage components $v_{i d}^{*}, v_{i q}^{*}$ are obtained by adding the measured network voltages $v_{n d}$ and $v_{n q}$, to the above control signals.

Although model derivation and treatment are in the synchronous $\mathrm{d}-\mathrm{q}$ frame, implementation of the control system may not need a phase-locked loop (PLL) [32] for estimating the angle of the network voltage vector, as active and reactive power are both DC quantities, calculated in either the stationary or in the synchronous reference frames. Therefore, feedback power measurement is directly provided through calculations based on 3 -phase direct voltage and current measurements at the PCC without a need for reference frame conversion. The control voltage components, $v_{i d}^{*}, v_{i q}^{*}$, can be converted to the stationary frame for the VSC's PWM modulator using the following conversion process which is based on the updated per-unit matrix of the $\alpha-\beta$ voltage components, 
$\underbrace{\left[\begin{array}{c}v_{i \alpha}^{*}(k T) \\ v_{i \beta}^{*}(k T)\end{array}\right]}_{\text {for PWM }}=\frac{1}{\left|\vec{v}_{n \alpha \beta}\right|}\left[\begin{array}{cc}v_{n \alpha}(k T) & -v_{n \beta}(k T) \\ v_{n \beta}(k T) & v_{n \alpha}(k T)\end{array}\right]\left[\begin{array}{l}v_{i d}^{*}(k T) \\ v_{i q}^{*}(k T)\end{array}\right]$.

Having the power controller structure as in Fig. 5, current variables or states are eliminated from the control process, enabling direct links between this controller and other subsystem outputs through its power references, as demonstrated in section 4. Such subsystems could be SI and frequency-droop (FD) controllers, which can be designed to produce active power demands, represented for the VSC system as,

$P_{n}^{*}(k T)=P_{\omega}^{*}(k T)=P_{\omega c}^{*}(k T)+P_{S I}^{*}(k T)$,

where, $P_{\omega}^{*}(k T)$ is the sum of two power demand components; $P_{\omega c}^{*}(k T)$ from the FD controller and $P_{S I}^{*}(k T)$ from the SI controller. To prevent the converter currents from exceeding their maximum limits in cases of balanced voltage sag within the local network, the power controller can be preceded by power de-rating functions which limit the power references, according to the magnitude of the network voltage-vector, using (2).

\subsection{Power controller tests and analysis}

To assess the performance of the proposed IMPC, time stepresponse tests have been performed by simulation for the VSCPG system under grid-connection with different operating conditions. In particular, the results shown in Fig. 7 (upper graph) demonstrate clearly the effectiveness of the IMPC when compared with the response of the CPC (middle graph) under the same test conditions; the proposed IMPC almost neutralizes power cross-coupling and removes the dead-time after 1s when the power reference was applied. Ideally, the power response of the IMPC would not have the lag of about $1 \mathrm{msec}$ observed in Fig. 7. This is caused by the measured power being pre-filtered to prevent changes in $P_{n}^{*}$ and $Q_{n}^{*}$ demands from being too large over one time-step. The observed lag, which has no impact on power variable decoupling, is not so large as to be intolerable. It is worth noting that whilst the CPC is sensitive to reduction in parameter $K_{i}$ (see subsection 3.3), the IMPC is insensitive to such variation for steady-state error and power cross-coupling reduction. As a result, the IMPC provided a step-response time of around $1 \mathrm{~ms}$ with no cross-coupling between active and reactive power and no sensitivity to variation in the proportional gain. Theoretically, the IMPC should eliminate cross-coupling between the active and reactive power at all stages of the power response, but due to modelling errors and approximation, an almost negligible power cross-coupling transient occurs. The extent of this cross-coupling is related to inaccuracies in the model when implemented in digital form. This controller results in power responses that are faster than those obtained through a synchronous-current controller (SCC) with an outer-loop power controller (OLPC) [40] [10], see Fig. 7 (bottom graph).

\section{Speed support with synthetic inertia via the VSC- PG}

DEGs are based on synchronous electrical machines where kinetic energy is stored in the rotating mass. However, exchanging this energy with other loads and sources is only possible to a limited extent to ensure that the generator speed/frequency stays within the allowed limits. To respond to transient loads the DEG utilises the engine governor to regulate its speed within these limits by varying the fuel injection, but the governor and engine response may not be sufficient to prevent frequency excursions from exceeding the limits. In comparison, a VSC-PG can be designed with a much faster power response, which compensates for any sudden change in load within the same isolated electrical network, to avoid noticeable variation in the DEG speed or network frequency.

The DEG can be utilized with three main control modes: isochronous, power-control and speed/FD control modes [33]. With the recent advancements in VSC systems and their control methods for high-power applications, VSC-based power
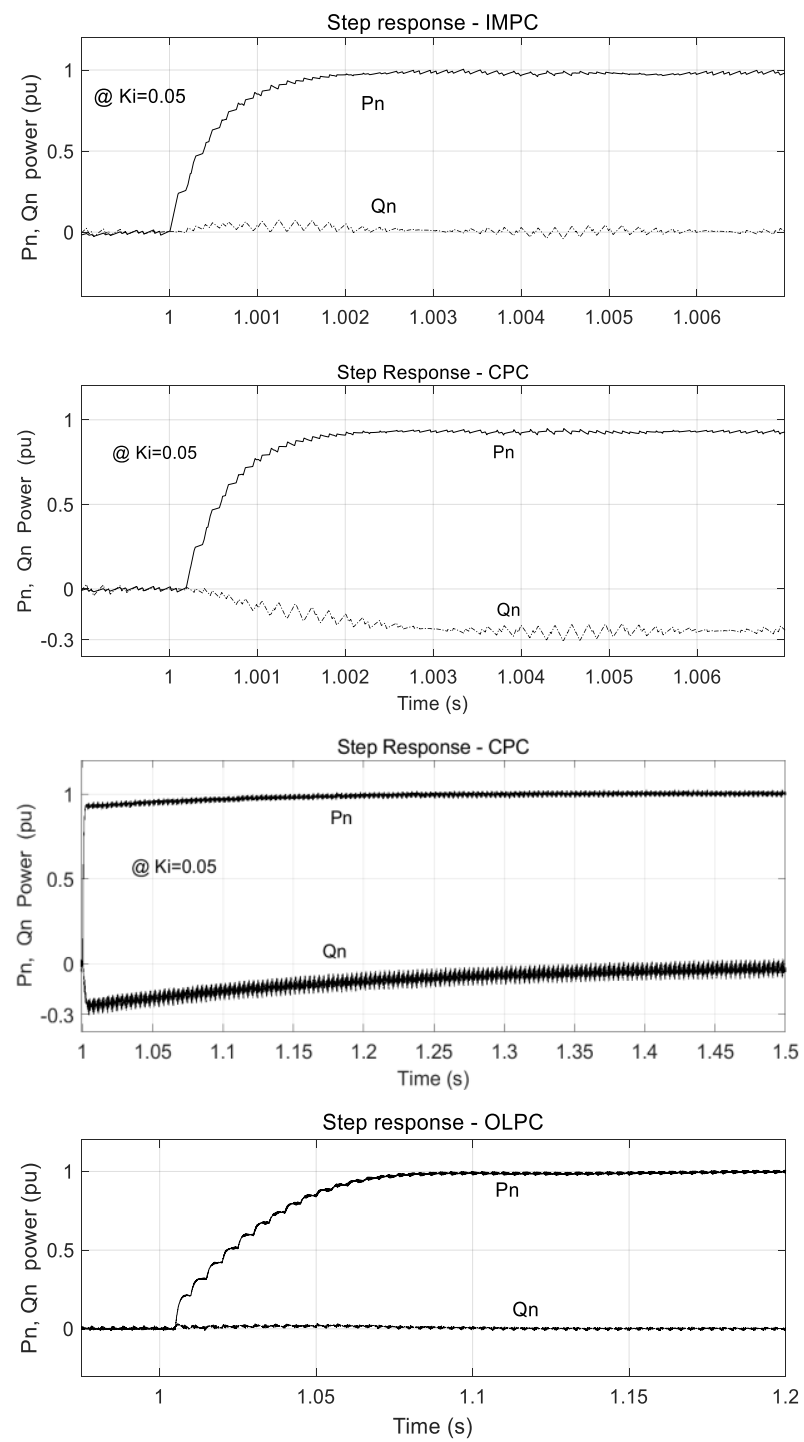

Fig. 7. (top graph): power step-response of the proposed IMPC, (middle graphs): power step-response of the CPC, (bottom graph): power step-response of the OLPC.

systems can now be controlled to emulate the operation of synchronous generators for all of these modes [34]. The factors influencing the speed (or the electrical angular frequency) of the rotating generator around its synchronous speed can be 
described, in general, with the help of the following simplified electromechanical relationship [35],

$J \omega_{m} \frac{d \omega_{m}}{d t}=P_{a} ;$

$P_{a}=\left(P_{m}(t)-P_{\text {loss }}(t)-P_{g}(t)\right)$,

where, $\omega_{m}$ is the generator speed, $P_{a}$ is the accelerating or decelerating power, $P_{m}(t)$ is the mechanical power supplied by the prime mover, $P_{g}(t)$ is the generator's electrical output power, $P_{\text {loss }}(t)$ represents the mechanical and electrical losses, and $J$ is the generator's moment of inertia. From this relationship, it is evident that there are two key variables affecting the generator frequency; the mechanical power and the electrical output power. In other words, the imbalance between the generator's mechanical power and the load (or the output electrical power) is the main factor that creates any speed/frequency deviation. When there is an electrical load change, the governor of the prime mover of the generating unit regulates the unit's speed by changing the mechanical power. In this context, it is possible to support frequency regulation of the DEG under isolated operation by letting the VSC-PG (incorporating an energy storage bank) provide a SI and share the load with the DEG to reduce the effect of transient events on the network frequency [36]-[37]. Based on the above electromechanical model, the concept of SI provision by the VSC, equivalent to that of the DEG, can be developed as follows. Equation (28) demonstrates a non-linear relationship between the generator speed and power as,

$\frac{d \omega_{m}}{d t}=\frac{P_{a}}{J \omega_{m}}=f\left(P_{a}, \omega_{m}\right)$.

By linearizing this relationship around the nominal speed, $\omega_{m s}$, or the operating-point, it becomes,

$\frac{d \Delta \omega_{m}}{d t} \approx \frac{1}{J \omega_{m s}} \Delta P_{a}-\frac{P_{a}^{o p}}{J \omega_{m s}^{2}} \Delta \omega_{m}$.

When speed equals the synchronous speed, $P_{a}^{o p}=0$, and the last term in (29) can be dropped, leading to the following linearized power-speed relationship,

$\frac{d \omega_{m}}{d t} \approx \frac{\Delta P_{a}}{J \omega_{m s}}$.

To oppose speed changes around the nominal operating-point, the SI power will be equal to $\Delta P_{a}$ but of the opposite sign, i.e. $P_{S I}^{*}=-\Delta P_{a}$, which can be combined with the FD power and implemented by the VSC for frequency support, as illustrated in Fig.8. When moving to a neighbouring operating-point due to a speed change, the speed can be updated regularly within (30) as it is changing slowly with respect to the system's electrical power dynamics. A new switching mechanism has been added to the conventional SI provider, [38], which only activates the action of the SI when the speed diverges from the nominal value according to the following conditions,

$\begin{aligned} & {\left[\left(\omega_{m}^{*}-\omega_{m}\right) \cdot \frac{d \omega_{m}}{d t}\right]} \\ & \text { or } \\ & {\left[\left(\omega_{m}^{*}-\omega_{m}\right) \cdot \Delta P_{a}\right]}\end{aligned}<0\left\{\begin{array}{l}\text { True } \rightarrow \text { SW2: Closed } \\ \text { False } \rightarrow \text { SW2:Open }\end{array}\right.$,
With this mechanism, the SI action will not impede the change in speed when approaching its set-point value. The switch, SW2, and its conditions, as shown in Fig. 8, controls the activation of the SI provider. For implementation, it can be difficult to realise the differentiation function, $\boldsymbol{D}$, unless the noise and humming superimposed on the speed signal are properly attenuated. Therefore, the differential term of the SI provider, $\boldsymbol{D}$, has been realised in combination with a first-order low-pass filter LPF that is necessary to mitigate noise effects when the speed signal is differentiated. This combination can be expressed in the Sdomain as follows,

$\underbrace{\frac{1}{1+\tau_{f} s}}_{\mathrm{LPF}} \cdot \underbrace{s}_{\boldsymbol{D}}=\frac{1}{\tau_{f}}\left(1-\frac{1}{1+\tau_{f} s}\right)$.

The realisation of the right hand-side term yields better results using a standard filter where, $\tau_{f}=1 / 2 \pi F_{c}$ and $F_{c}$ is the cut-off frequency of the filter, which was chosen to be equal to half of

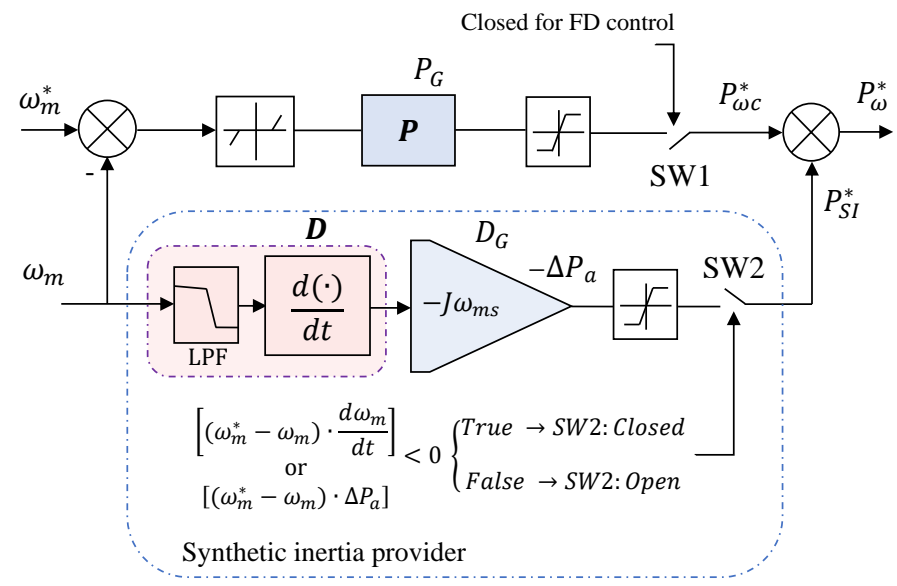

Fig. 8. Block diagram of the of the proposed SI provider with switching mechanism including FD controller

the generator nominal frequency. The output of this combined function is then scaled by $-J \omega_{m s}$ to give the required SI power, where $J$ can take any value to emulate the inertia of a rotating generator of any size, or even to emulate the total inertia of a number of synchronised generators. For the case-study presented in the paper, $J$ was given the same value as the DEG, as given in Appendix 1. It should be noted that to avoid multiple switching of SW2 at speed-error zero-crossing, or when $\omega_{m}$ is very close to its set-point, a hysteresis function with a width greater than the maximum peak-to-peak ripple in the speed signal can be applied to the speed-error signal $\left(\omega_{m}^{*}-\omega_{m}\right)$ before using it in the term $\left[\left(\omega_{m}^{*}-\omega_{m}\right) \cdot \Delta P_{a}\right]$. Otherwise, this ripple may indirectly lead the DEG governor controller to behave in an undesirable manner. The new SI switching mechanism has been tested and compared with the traditional method given in [39]-[41] in terms of DEG frequency-support performance in isolated operation of the microgrid, as discussed in the next section. 


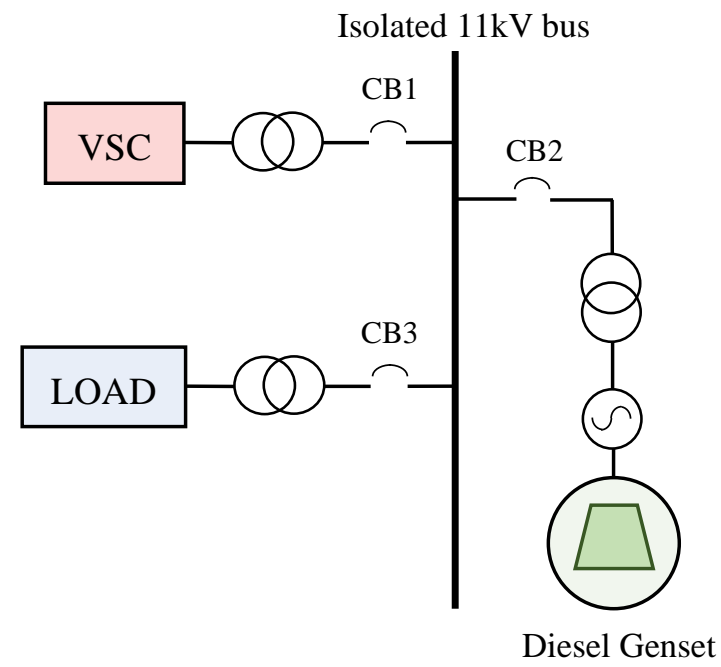

Fig. 9. Block diagram of the isolated-microgrid components connected through an $11 \mathrm{kV}$ bus

\section{Simulation results and analysis}

The proposed IMPC method and SI technique, with switching mechanism, have been tested for the microgrid configuration given in Fig. 9. All of the grid components are connected to an isolated $11 \mathrm{kV}$ bus through an $11 \mathrm{kV} / 415 \mathrm{~V}$ transformers and circuit breakers, $\mathrm{CBn}$. The load is controllable so that load steps can be applied at any time instance for testing the frequencyresponse when utilising SI provision and FD control. The physical power components of the microgrid have been built using the continuous-time simulation blocks provided by the Power System Toolbox of the Matlab/Simulink environment.

\subsection{Testing the performance of the SI provider}

For comparison with the proposed SI power demand component, in terms of performance, the under-frequency controller (UFC) in [40], which uses both SI and FD channels in parallel, is considered. The frequency activation limit of the UFC is first set to $49.99 \mathrm{~Hz}$ for dynamic continuous operation within the isolated low-inertia microgrid. The structures of the system in Fig. 8 and the UFC are similar regarding the FD channel but different in that the proposed SI power demand component includes a switching mechanism represented by SW2 and its related conditions, where the SI is only applied to support generator frequency when needed. Fig. 10(a) shows the simulation results for the speed or frequency-response when the DEG is suddenly loaded with an additional $0.1 \mathrm{pu}$ load step at time 13s. Trace 1 of the top graph represents the response without activating the SI power demand component with frequency nadir approaching the $0.98 \mathrm{pu}$, whilst trace 2 demonstrates the improvement when using the SI power demand component in addition to a considerable reduction in the ROCOF, from $9.6 \mathrm{~Hz} / \mathrm{s}$ to $4.3 \mathrm{~Hz} / \mathrm{s}$. By introducing SW2, trace 3 clearly shows the enhancement in the response after the nadir as the speed moves closer to the nominal line without overshoot or with a shorter settling-time. The performance of the SI with the switching mechanism, in terms of frequencyresponse support, is clearly demonstrated. The SI switching mechanism blocks the application of the SI power demand component when the speed approaches its nominal or set point value, therefore, the SI derivative action will not impede the DEG speed governor from regulating the speed/frequency.The second graph of Fig. 10(a) shows the corresponding power traces produced by the VSC as demanded by the SI power demand component. It should be noted that the VSC power contains the PWM switching component as it is measured at a point before the grid's high-pass power filter. The proposed SI power demand component (with switching mechanism) does not demand power whilst the speed approaches its set point. For the case when the frequency support system includes an FD controller, Fig. 10(b) shows the response under the same condition as in Fig. 10(a) with gain, $D_{G}=10^{5} / \pi$. Although the effect of using the FD controller on the response is quite dominant in increasing the frequency nadir above $0.99 \mathrm{pu}$, the SI switching mechanism continues to present a slight improvement as demonstrated by trace 3 in Fig. 10(b). However, the FD controller demands more energy or average power to be produced by the VSC which has a limited VA power that may be needed for other purposes. As the UFC system in [40] was implemented by an OLPC, it is worthwhile to compare its performance with the proposed system using the IMPC. Both systems were simulated and the speed/frequency responses in Fig. 11 are obtained under the same conditions considered for the previous results. In the first graph of Fig. 11(a), trace 1 represents the response when using the proposed SI power demand component (with switching mechanism) and implementing its output power-demand utilising the IMPC, whilst trace 2 represents the response when using the UFC and implementing its output through the OLPC. The effect of the delay imposed by the OLPC has caused the ROCOF before the nadir to be steeper, with almost double that of trace 1 . In comparison, the results obtained when implementing the output using the IMPC demonstrate better performance in terms of response settling-time too. Further favourable comparison is observed when the deadband of the UFC is set to $[49.8 \mathrm{~Hz}$ to $50.2 \mathrm{~Hz}$ ], [41], which resulted in trace 3 that demonstrates a lower nadir and slight increase in the ROCOF. The traces in the second graph represent the corresponding power generated by the VSC for each case. By adding the FD control function to the proposed SI power demand component, the top graph of Fig. 11(b) shows far better improvement in speed response compared with that of the UFC (using the previous deadband setting) but with considerably higher power and energy demands, which can be regarded as a disadvantage for VSC power availability. The corresponding SI power traces are illustrated in the bottom graph of Fig. 11(b).

For further assessment, a quantitative approach is also followed for evaluating the performance of the SI power demand component with the switching mechanism. Table 1 provides a quantitative comparison of the proposed SI power demand component with the UFC in terms of frequency-response features when the DEG is subjected to a $0.1 \mathrm{pu}$ load step. It can be noticed from the table that the SI power demand component with the switching mechanism resulted in a lower settling-time accompanied with low variation in frequency and angle lag when compared with the SI power demand component without the switching mechanism. The latter produced a big difference 

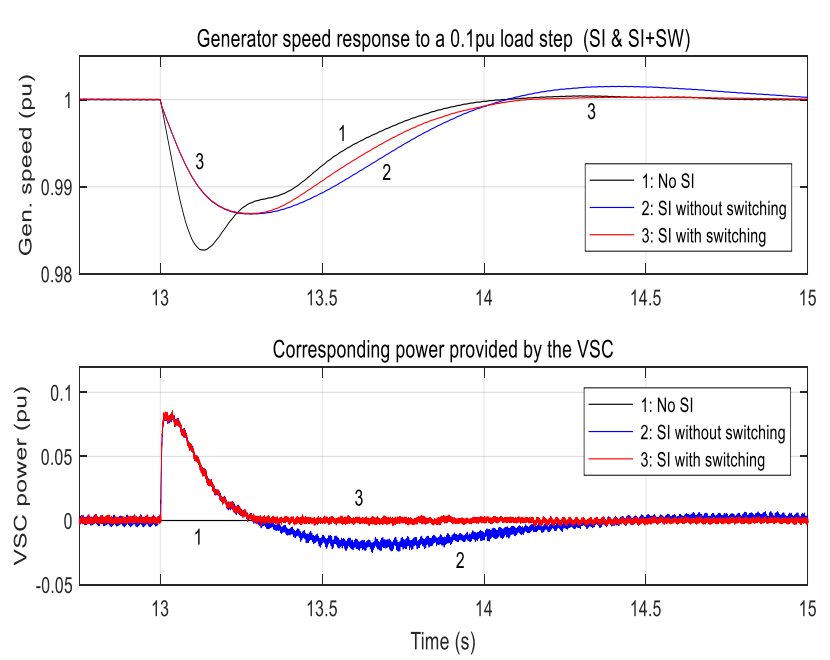

(a)
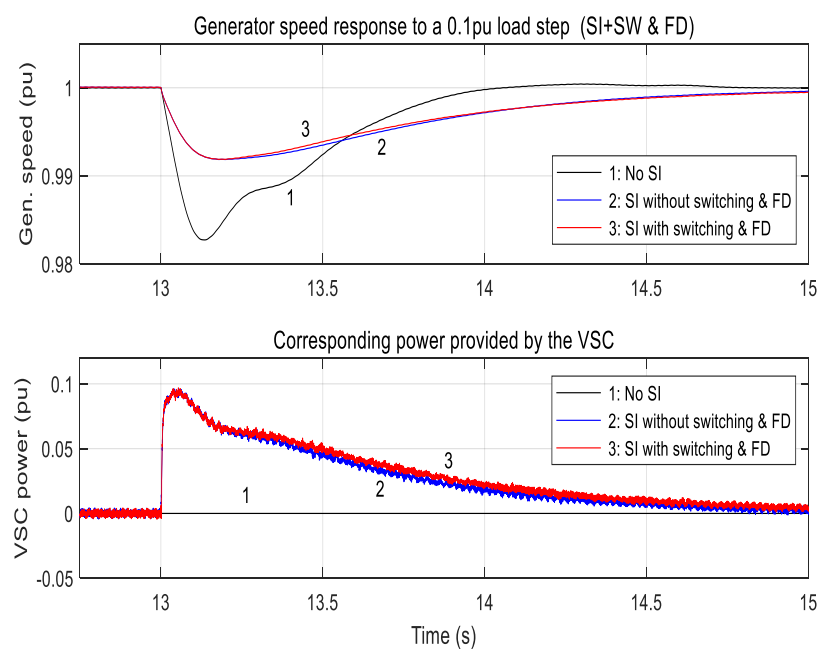

(b)

Fig. 10. Frequency-response performance under 0.1pu load step

(a) top graph: trace 1: without SI; trace 2: with standard SI; trace 3: SI with switching mechanism. bottom graph: the corresponding SI power.

(b) top graph: trace 1: without SI; trace 2: with SI \& FD control; trace 3: SI with switching mechanism \& FD control. bottom graph: the corresponding power.

in settling-time within a speed-band equal to 0.002 [pu], or $\pm 10 \%$ of $\pm 1 \%$ deviation around the reference speed. The last two rows of the table give the frequency nadirs and angle lags produced when utilizing the proposed SI power demand component augmented with the FD control and implemented by the IMPC, and when the UFC is implemented using the OLPC, respectively. The difference in performance between the two methods is clear. The inclusion of the FD considerably improved the frequency nadir but the implementation of the UFC power using OLPC caused an extra angle lag due to the delay introduced by the OLPC and the deadband of the UFC. The fourth column of Table 1 provides the recorded settlingtimes of the speed responses for all the SI methods for
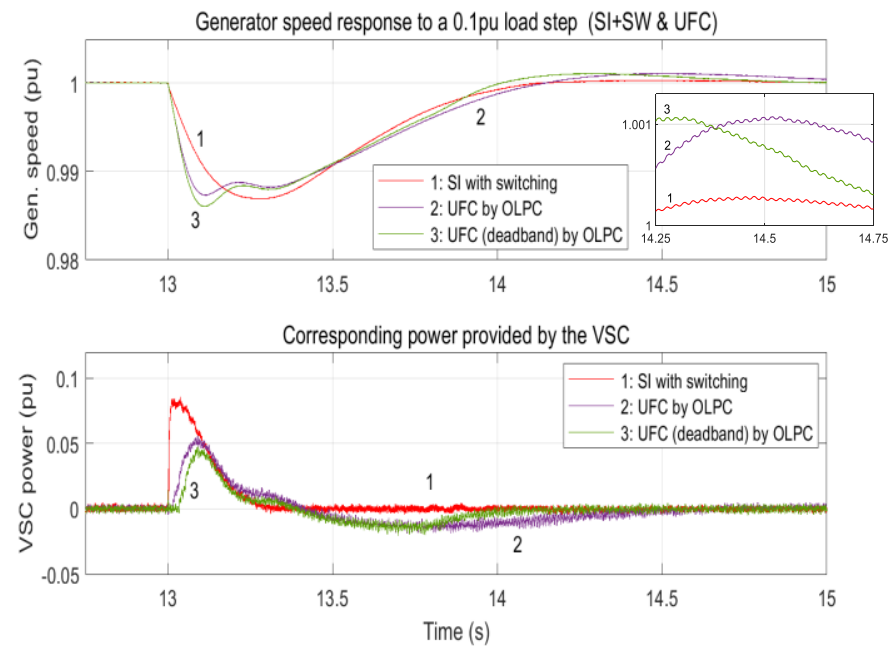

(a)
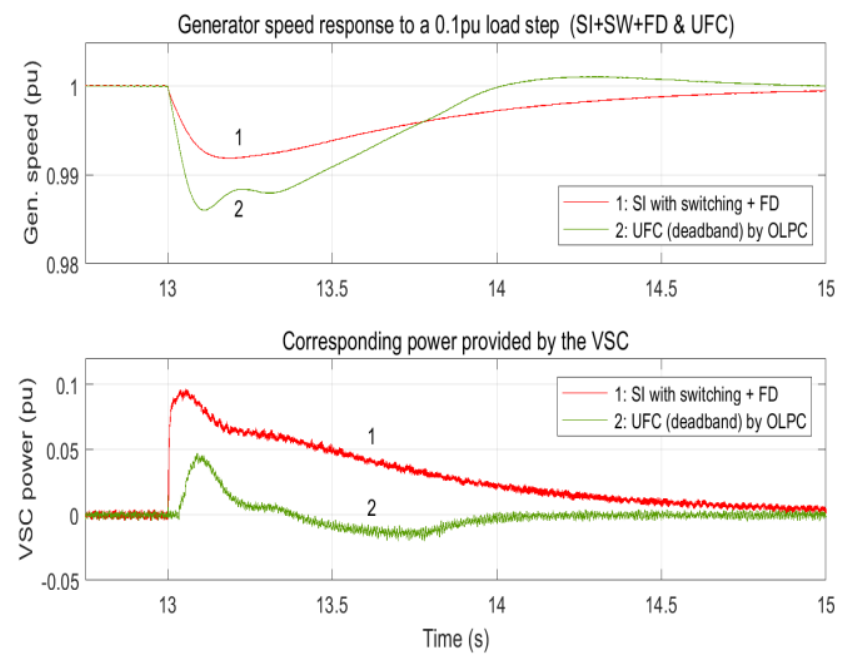

(b)

Fig. 11. Frequency-response performance under 0.1pu load step

(a) top graph: trace 1: SI with switching mechanism implemented by IMPC; trace 2 \& 3: UFC implemented by OLPC. bottom graph: the corresponding power.

(b) top graph: trace 1: SI with switching mechanism \& FD control by IMPC; trace 2: UFC (deadband) by OLPC. bottom graph: the corresponding power.

comparison. The SI method (with the switching mechanism) implemented by the IMPC yielded the shortest settling-time when compared with the other SI and power control methods. The advantage of implementing the IMPC as a direct power controller for SI provision using the proposed switching mechanism is evident.

\section{Conclusion}

This paper presents a newly developed IMPC based on a dynamic power model for a VSC-based power generator connected to a microgrid PCC and to a DC-link powered by a battery-bank. The paper also proposed an approach to providing SI with a switching mechanism, which resulted in an improved 
frequency-response performance when compared with the standard method. By implementing SI using IMPC, a clear improvement in response performance is achieved in comparison with SI using UFC by an OLPC. The results obtained through a detailed systematic simulation of the proposed IMPC and SI have demonstrated the effectiveness of these methods in supporting the regulation of the DEG speed/frequency within an isolated microgrid with low-inertia rotating generators.

Table 1 Quantitative comparison of the proposed SI provider with a standard UFC in terms of frequency-response features, caused angle lag and settling-time (within a speed-band equal to 0.002 [pu], i.e. $\pm 10 \%$ of $\pm 1 \%$ deviation around the reference speed) when the DEG is subjected to a $0.1 \mathrm{pu}$ load step.

\begin{tabular}{|l|c|c|c|}
\cline { 2 - 4 } & $\begin{array}{c}\text { Frequency } \\
\text { nadir [pu] }\end{array}$ & $\begin{array}{c}\text { Caused } \\
\text { angle lag } \\
\text { [rad] }\end{array}$ & $\begin{array}{c}\text { Settling } \\
\text { time } \\
{[\mathrm{sec}]}\end{array}$ \\
\hline \hline Without SI & 0.982 & 1.191 & 0.8761 \\
\hline $\begin{array}{l}\text { SI without switching } \\
\text { (as standard) }\end{array}$ & 0.9869 & 1.123 & 1.7035 \\
\hline $\begin{array}{l}\text { SI with switching } \\
\text { mechanism }\end{array}$ & 0.9869 & 1.121 & 0.9717 \\
\hline \hline $\begin{array}{l}\text { SI with switching \& } \\
\text { FD control } \\
\text { implemented by the } \\
\text { IMPC; } G_{p}=10^{5} / \pi\end{array}$ & 0.9919 & 1.075 & 1.6116 \\
\hline $\begin{array}{l}\text { UFC with frequency } \\
\text { deadband as in [40] } \\
\text { implemented by the } \\
\text { OLPC }\end{array}$ & 0.9861 & 1.1267 & 1.3956 \\
\hline
\end{tabular}

\section{Acknowledgements}

F. Hardan gratefully acknowledges the sponsorship granted by Newcastle University for his academic fellowship, and the award of this fellowship through the CARA programme in London, UK.

\section{Appendix 1}

The specification and parameters of the VSC-Based power generator's components utilized throughout this study:

- the bi-polar DC-link: $\pm 550 \mathrm{~V}$

- the linking 3-phase inductance: $L_{l}=337 \mu \mathrm{H}, R_{l}=2.6 \mathrm{~m} \Omega$

- the 3-phase $\Delta / Y$ transformer: $0.63 \mathrm{MVA}, 50 \mathrm{~Hz}$

- $\Delta$ windings: $V_{l l ~ r m s}=400 \mathrm{~V}, R_{1}=0.64 \mathrm{~m} \Omega, L_{1}=81.5 \mu \mathrm{H}$

- Y windings: $V_{l l ~ r m s}=11 \mathrm{kV}, R_{2}=0.4 \Omega, L_{2}=50.9 \mathrm{mH}$

- network-connection parameters:

(from the linking inductance and the transformer parameters):

- total inductance: $L_{n}=0.5 \mathrm{mH}$

- total resistance: $R_{n}=7.93 \mathrm{~m} \Omega$

- time-constant: $\tau_{n}=\frac{L_{n}}{R_{n}} \cong \frac{1}{16} \mathrm{~s}$
The specification and parameters of the DEG and its transformer utilized throughout this study:

- generator inertia constant and speed: $H=0.272 s$, $\omega_{m}=1500 \mathrm{rpm}$

- generator moment of inertia: $J=20 \mathrm{~kg} . \mathrm{m}^{2}$

- the 3-phase $\Delta / Y$ transformer: $1 \mathrm{MVA}, 50 \mathrm{~Hz}$

- $\Delta$ windings: $V_{l l ~ r m s}=400 \mathrm{~V}, R_{1}=0.32 \mathrm{~m} \Omega, L_{1}=40.7 \mu \mathrm{H}$

- Y windings: $V_{l l ~ r m s}=11 \mathrm{kV}, R_{2}=0.242 \Omega, L_{2}=30.8 \mathrm{mH}$

\section{References}

[1] Hu, J., Shan, Y., Xu, Y., Guerrero, JM.: 'A coordinated control of hybrid ac/dc microgrids with PV-wind-Battery under variable generation and load conditions', Electrical Power and Energy Systems, 2019, 104, pp. 583-592

[2] Hardan, F.: 'An efficient dynamic control of modern renewable distributed generators for intentional islanding operation'. Proc. Int. Conf. IET-DPSP, Birmingham, UK, April 2012, pp. 23-28

[3] Mesbahi, N., Ouari, A., Abdeslam, DO., Djamah, T., Omeiri, A.: 'Direct power control of shunt active filter using high selectivity filter (HSF) under distorted or unbalanced conditions', Electric Power Systems Research, 2014, 108, pp. $113-123$

[4] Serpa, LA., Ponnaluri, S., Barbosa, PM., Kolar, JW.: 'A Modified Direct Power Control Strategy Allowing the Connection of Three-Phase Inverters to the Grid through LCL Filters', IEEE Transaction on Industry Applications, 2007, 43, (5), pp. 1388-1400

[5] Hu, J., Shang, L., He, Y., Zhu, ZQ.: 'Direct Active and Reactive Power Regulation of Grid-Connected DC/AC Converters Using Sliding Mode Control Approach', IEEE Trans on Power Electronics, 2011, 26, (1), pp. 210-222

[6] Gui, Y., Kim, C., Chung, CC., Guerrero, JM., Guan, Y., Vasquez, JC.: 'Improved Direct Power Control for GridConnected Voltage Source Converters'. IEEE Trans on Industrial Electronics, 2018, 65, (3), pp. 8041-8051

[7] Eloy-Garcia, J., Arnaltes, S., Rodriguez-Amenedo, JL.: 'Direct power control of voltage source inverters with unbalanced grid voltages', IET Power Electronics, 2008, 1, (3), pp. $395-407$

[8] Friedland, B.: 'Control System Design - An Introduction to State-Space Methods' (Dover Publications Dover ed., Mineola, New York, 2005)

[9] Springob, L., Holtz. J., 'High-Bandwidth Current Control for Torque-Ripple Compensation in PM Synchronous Machines', IEEE Transaction on Industrial Electronics, 1998, 45 (5), pp. 713-721

[10] Zhao, S., Shao, B.: 'An analytical method suitable for revealing the instability mechanism of power electronics dominated systems', Electrical Power and Energy Systems, 2019, 109, pp. 269-282

[11] Unamuno, E., Barrena, JA.: 'Hybrid ac/dc microgrid-Part I: Review and classification of topologies', Renewable and sustainable Energy Reviews, 2015, 52, pp. 1251-1259

[12] Giaouris, D., Papadopoulos, AI., Patsios, C., Walker, S., Ziogou, C., Taylor, P., Voutetakis, S., Papadopoulou, S., Seferlis, P.: 'A system approach for management of microgrids 
considering multiple energy carrier, stochastic loads, forcasting and demand side response', Applied Energy, 2018, 226, pp. 546-559

[13] Unamuno, E., Barrena, JA.: 'Hybrid ac/dc microgrid-Part II: Review and classification of control strategies', Renewable and sustainable Energy Reviews, 2015, 52, pp. 1123-1134

[14] Greenwood, DM., Lim, KY., Patsios, C., Lyons, PF., Lim, YS., Taylor, PC.: 'Frequency response services designed for energy storage', Applied Energy, 2017, 203, pp. 115-127

[15] Tielens, P., Hertem, DV.: 'The relevance of inertia in power systems', Renewable and Sustainable Energy Reviews, 2016, 55, pp. 999-1009

[16] Wilson, D., Yu, J., Al-Ashwal, N., Heimission, B., Terzija, V.: 'Measuring effective area inertia to determine fast-acting frequency response requirements', Electrical Power and Energy Systems, 2019, 113, pp. 1-8

[17] Al-Masood, N., Modi, N., Yan, R.: 'Low Inertia Power Systems: Frequency Response Challenges and a possible Solution'. Proc. Australasian Universities Power Engineering Conf. (AUPEC), Brisbane, QLD, Australia, Sept 2016, DOI: 10.1109/AUPEC.2016.7749335

[18] Gloe, A., Jauch, C., Cracium, B., Winkelmann, J.: 'Continuous provision of synthetic inertia with wind turbines: implications for the wind turbine and for the grid', IET Renewable Power Generation, 2019, 13, (5), pp. 668-675

[19] Duckwitz, D., Fischer, B.: 'Modeling and Design of df/dtBased Inertia Control for Power Converters', IEEE Journal of Emerging and Selected Topics in Power Electronics, 2017, 5, (4), pp. 1553-1564

[20] Mehrasa, M., Pouresmaeil, E., Soltani, H., Blaabjerg, F., Calado, MRA., Catalao, JPS.: 'Virtual Inertia and Mechanical Power-Based Control Strategy to Provide Stable Grid operation under high renewable Penetration', Applied Sciences, 2019, 9, 1043, pp. 1-16

[21] Mehrasa, M., Sharifzadeh, M., Al-Haddad K.: 'A Droop Based-Control Strategy of Stand-Alone Single-Phase Converters for microgrid Applications'. 44th Annual Conference of the IEEE Industrial Electronics Society (IECON), Washington, DC, USA, Dec 2018, DOI: 10.1109/IECON.2018.8591139

[22] Mondal, SK., Bose, BK., Oleschuk, V., Pinto, JOP.: 'Space Vector Pulse Width Modulation of Three-Level Inverter Extending Operation into Overmodulation Region', IEEE Transaction on Power Electronics, 2003, 18, (2), pp. 604-611

[23] Rodríguez, J., Bernet. S., Wu. B., Pontt. JO., Kouro. S.: 'Multilevel Voltage-Source-Converter Topologies for Industrial Medium-Voltage Drives', IEEE Transactions on Industrial Electronics, 2007, 54, (6), pp. 2930-2945

[24] Chavesa, M., Margatoa, E., Silvaa, JF., Pintoa, SF., Santanaa, J.: 'HVDC transmission systems: Bipolar back-toback diode clamped multilevel converter with fast optimumpredictive control and capacitor balancing strategy', Electric Power Systems Research, 2011, 81, pp. 1436-1445

[25] Grainger, JJ., William, WD., Chang, GW.: 'Power System Analysis' (McGraw-Hill Education, New York, 1994, 2nd edn. 2015)

[26] Akagi, H.: 'Active Filters for Power Conditioning', in Grigsby Leonard Lee, Ed., Boca Raton, FL. (Ed.): 'Power
Systems' (CRC Press, Taylor \& Francis Group, 2007, 2nd edn.), pp. 394-396

[27] Shamma, JS., Athens, M.: 'Garanteed properties of gainscheduled control for linear parameter-varying plants', Automatica, 1991, 27, (3), pp. 559-564

[28] Leith, DJ., Leithead, WE.: 'Survey of gain-scheduling analysis and design', Inter. Journal of Control, 2000, 73, (11), pp. 1001-1025

[29] Ogata, K.: 'Modern Control Engineering' (Pearson, Boston, 5th edn. 2010)

[30] Barros, CPB., Barros, PR., Neto, JSR.: 'Loop Shaping for PID Controller Design Based on Time and Frequency Specification', IFAC PapersOnLine, 2018, 51-4, pp. 592-597

[31] Devasia, S.: 'Should model-based inverse inputs be used as feedforward under plant uncertainty?', IEEE Trans on Automatic Control, 2002, 47, (11), pp. 1865-1871

[32] Jaalam, N., Rahim, NA., Bakar, AHA., Tan, C., Haidar, A.: 'A comprehensive review of synchronization methods for grid-connected converters of renewable energy source', Renewable and Sustainable Energy Reviews, 2016, 59, pp. 1471-1481

[33] Boldea, I.: 'Synchronous Generators' (CRC Press, Taylor \& Francis Group, Boca Raton, 2nd edn. 2015)

[34] Serban, I., Ion, CP.: 'Microgrid control based on a gridforming inverter operating as virtual synchronous generator with enhanced dynamic response capability', Electrical Power and Energy Systems, 2017, 89, pp. 94-105

[35] Glover, JD., Sarma, MS., Overbye, TJ.: 'Power System Analysis and Design' (Cengage Learning, Stamford, CT, 5th edn. 2012)

[36] Tielens, P., Van Hertem, D.: 'The relevance of inertia in power systems', Renewable and Sustainable Energy Review, 2016, 55, pp. 999-1009

[37] Vokony, I.: 'Effect of inertia deficit on power system stability - synthetic inertia concepts analysis'. Proc. Int. Conf. 6th International Youth Conference on Energy (IYCE), Budapest, Hungary, Aug 2017, DOI: 10.1109/IYCE.2017.8003725

[38] Duckwitz, D., Fischer, B.: 'Modeling and Design of df/dtBased Inertia Control for Power Converters', IEEE Journal of Emerging and Selected Topics in Power Electronics, 2017, 5, (4), pp. 1553-1564

[39] Taczi, I.: 'Enhancing Power System Frequency Stability with Synthetic Inertia'. Proc. Int. Conf. IEEE EUROCON 2017 -17th International Conference on Smart Technologies, Ohrid, Macedonia, Aug 2017, DOI: 10.1109/EUROCON.2017.8011254

[40] Liu, H., Chen, Z.: 'Contribution of VSC-HVDC to frequency regulation of power systems with offshore wind generation', IEEE Transaction on Energy Conversion, 2015, 30, (3), pp. 918-926.

[41] Gonzalez-Longatt, F. M.: 'Activation Schemes of Synthetic Inertia Controller on Full Converter Wind Turbine (Type 4)'. Proc. Int. Conf. IEEE Power \& Energy Society General Meeting, Denver, CO, USA, July 2015, DOI: 10.1109/PESGM.2015.7286430 